\title{
An Unsteady Magnetohydrodynamics Flow of Bingham Fluid with Hall Effect of Heat Transfer
}

\author{
Pudhari Srilatha, J. Suresh Goud, B. Raju, S. Devaraj
}

\begin{abstract}
In this paper we investigated an unsteady magnetohydrodynamics flow of Bingham fluid with Hall Effect of heat transfer. Partial differential equations are simplified to higher order differential equations. MATLAB integrated bvp4c digital solver for velocity and temperature solves a set of nonlinear ordinary differential equations. The graphs show the effect of different parameters of velocity and temperature.
\end{abstract}

Keywords: Bingham fluid, Magnetohydrodynamics, Hall Effect, Heat Transfer.

\section{INTRODUCTION}

Bingham fluid is a viscous fluid and non-Newtonian fluid that meets the flow limit and must exceed this flow limit before the fluid flows. The name was created by E. C. Bingham after the introduction of his mathematical model in 1916. Toothpaste jumps into the tube only after some pressure. Bingham fluids are used in various geological and mathematical materials for engineering, aerospace engineering and chemical technology. For example: mud, mud, cement, mud, grease, chocolate etc. Nigam, singh [1] investigated the thermal displacement between parallel plates is affected by the transverse magnetic field. R. Alpher [2] examined magnetic fluid flow between parallel plates in heat transfer. Walters K.[3]studied the development of nonNewtonian fluid mechanics. Astarita G.[4] studied three alternative approaches to developing governance equations. Walton Bittleston [5] studied Articulation of Bingham's plastic flow from a small eccentric circle. Attia, Kotb [6] examined heat wave occurring between two parallel lines in magnetic field. Attia, Transient [7] studied the heat transfer between the MHD flow and the two parallel plates depend on temperature and viscosity. Chamkha [8] investigated the unstable layer of particles is a circular magnetic flux for the transfer of fluid and heat in these channels and channels. Attia [9] studied the unstable flow of HMD and heat transfer between the powders in parallel plates with different physical properties. Hazem Ali Attia and sayed ahmed [10] examined hall effect on the flow and heat transfer of transient particles of MHD, the aspiration and injection of bingham fluid.

Revised Manuscript Received on December 30, 2019.

* Correspondence Author

Pudhari Srilatha, Institute of Aeronautical Engineering, Hyderabad, Telangana State, India.

J. Suresh Goud, Institute of Aeronautical Engineering, Hyderabad, Telangana State, India.

B. Raju, Institute of Aeronautical Engineering, Hyderabad, Telangana State, India.

S. Devaraj, Institute of Aeronautical Engineering, Hyderabad, Telangana State, India.

(C) The Authors. Published by Blue Eyes Intelligence Engineering and Sciences Publication (BEIESP). This is an open access article under the CC BY-NC-ND license (http://creativecommons.org/licenses/by-nc-nd/4.0/)
Chamkha [11] considered convection heat transfer and unstable MHD mass transfer are achieved by heat transfer and mass transfer by a heated semi perpendicular mobile plate, as well as by heat transfer and a semi perpendicular endothermic mobile plate. Ogulu, Amakiri and Mbeledogu [12] investigated the radiation heat transfer process, no instability wave convey or compressible fluid to flow into the plate to move vertically. Ramchandra Prasad, and Bhaskar Reddy [13] studied the effect of radiation convective heat and mass transfer by the HMD unstable semi-infinite vertical plate embedded in a porous medium for transmitting. Dass, Satapathy, Panda [14] considered under the influence of suction, the vibratory and thermal transmission energy as a result of heat transfer and mass transfer from the mass flow source is transmitted through a porous medium that is rare along the plate vertical. Ahmed $\mathrm{S}$, Khatun $\mathrm{H}$ [15] studied flow of the oscillating magnetic fluid in the porous blood vessels of flat suction and injection. Makinde, Chinyoka , Rundora [16] investigated the transient of a non-newtonian variable viscosity fluid undergoes an asymmetric porous saturated medium under the conditions of a convection profile. Rundora, Makinde [17] examined a porous channel filled with a medium is introduced by a flow of non-newtoinan non-reactive liquid under stationary and convective condition. Chuo-Jeng Huang [18] studied the effect of a uniform bladder/absorption on non-Newtonian convection without fluid in a vertical cone in porous media with thermal radiation and Soret/Dufour effects.

In view of the above discussion, the main objective of this work is to solve the unsteady flow of fluid to heat soil magnetohydrodynamics Bingham hall transfer. The effects can be extracted from the non-linear differential equation, which can be solved using MATLAB bvp4c with an integrated digital solver.

\section{MATHEMATICAL FORMULATION}

Study of viscous uncompressed conductive Bingham fluid in parallel plate channels defined by the original porous medium. The fluid moves with a uniform pressure gradient parallel to the channel plate, and a magnetic field with a uniform intensity gradient Ho, inclined at an angle oblique to the normal to the $\mathrm{xz}$ - plane, acts throughout the flow field. The fluid undergoes a constant change of pressure in the $\mathrm{X}$ direction at $\mathrm{t}=0$ and has a uniform suction up and a uniform lower Injection. 
A uniform magnetic field is ignored due to the assumption of a very small Reynolds number; therefore, it is assumed that this does not affect the magnetic field. Due to the Hall Effect, The $\mathrm{z}$ component of the speed is constant. Therefore, the fluid velocity vector is defined as

$$
\begin{aligned}
& v^{\prime}(y, t)=v^{\prime}(y, t) \overline{\mathbf{i}}+v_{0} \overline{\mathbf{j}}+w^{\prime}(y, t) \overline{\mathbf{k}} \\
& \rho \frac{D v^{\prime}}{D t}=\nabla \cdot\left(\mu \nabla v^{\prime}\right)-\nabla p+J \times B_{0} \\
& \rho \frac{\partial u^{\prime}}{\partial t}+\rho v_{0} \frac{\partial u^{\prime}}{\partial y}=-\frac{d p}{d x}+\frac{\partial}{\partial y}\left(\mu \frac{\partial u^{\prime}}{\partial y}\right) \\
& -\frac{\sigma B_{0}^{2} \operatorname{Sin}^{2} \alpha}{1+m^{2} \operatorname{Sin}^{2} \alpha}\left(u+m \operatorname{Sin} \alpha w^{\prime}\right)-\frac{v^{\prime}}{k} u^{\prime} \\
& ---(2) \\
& \rho \frac{\partial w^{\prime}}{\partial t}+\rho v_{0} \frac{\partial w^{\prime}}{\partial y}=\frac{\partial}{\partial y}\left(\mu \frac{\partial u^{\prime}}{\partial y}\right) \\
& -\frac{\sigma B_{0}^{2} \operatorname{Sin}^{2} \alpha}{1+m^{2} \operatorname{Sin}^{2} \alpha}\left(w^{\prime}-m \operatorname{Sin} \alpha u^{\prime}\right) \\
& -\frac{v^{\prime}}{k} w^{\prime} \\
& \rho c_{p} \frac{\partial T}{\partial t}+\rho c_{p} v_{0} \frac{\partial T}{\partial y}=k \frac{\partial^{2} T}{\partial y^{2}} \\
& +\mu\left[\left(\frac{\partial u^{\prime}}{\partial y}\right)^{2}+\left(\frac{\partial w^{\prime}}{\partial y}\right)^{2}\right] \\
& +\left(\frac{\sigma B_{0}^{2} \operatorname{Sin}^{2} \alpha}{1+m^{2} \operatorname{Sin}^{2} \alpha}-\frac{v^{\prime}}{k}\right)\left(u^{\prime 2}+w^{\prime 2}\right)
\end{aligned}
$$

Dimensionless quantities

$$
\begin{aligned}
& x^{*}=\frac{x}{h}, y^{*}=\frac{y}{h}, z^{*}=\frac{z}{h}, t^{*}=\frac{t U_{0}}{h}, \\
& w^{*}=\frac{w}{U_{0}}, p^{*}=\frac{p}{\rho U_{0}^{2}}, \theta=\frac{T-T_{1}}{T_{2}-T_{1}}, \mu^{*}=\frac{\mu}{K}
\end{aligned}
$$

By using non-dimensional quantities, equations

$$
\text { (2), (3) and (4) reduced to }
$$

$$
\begin{aligned}
& \frac{\partial u^{\prime}}{\partial t}+\frac{S}{\operatorname{Re}} \frac{\partial u^{\prime}}{\partial y}=-\frac{d p}{d x}+ \\
& \frac{1}{\operatorname{Re}}\left[\begin{array}{l}
\frac{\partial}{\partial y}\left(\mu \frac{\partial u^{\prime}}{\partial y}\right)-\frac{M^{2} \operatorname{Sin}^{2} \alpha}{1+m^{2} \operatorname{Sin}^{2} \alpha} \\
\left(u^{\prime}+m \operatorname{Sin} \alpha w^{\prime}\right)-\frac{v^{\prime}}{k} u^{\prime}
\end{array}\right]
\end{aligned}
$$

$$
\begin{aligned}
& \frac{\partial w^{\prime}}{\partial t}+\frac{S}{\operatorname{Re}} \frac{\partial w^{\prime}}{\partial y}=-\frac{d p}{d x} \\
& +\frac{1}{\operatorname{Re}}\left[\begin{array}{l}
\frac{\partial}{\partial y}\left(\mu \frac{\partial w^{\prime}}{\partial y}\right)-\frac{M^{2} \operatorname{Sin}^{2} \alpha}{1+m^{2} \operatorname{Sin}^{2} \alpha} \\
\left(w^{\prime}+m \operatorname{Sin} \alpha u^{\prime}\right)-\frac{v^{\prime}}{k} w^{\prime}
\end{array}\right]
\end{aligned}
$$

$$
\begin{aligned}
& \frac{\partial \theta}{\partial t}+\frac{S}{\operatorname{Re}} \frac{\partial \theta}{\partial y}=\frac{1}{\operatorname{Pr}} \frac{\partial^{2} T}{\partial y^{2}} \\
& +E c \mu\left[\left(\frac{\partial u^{\prime}}{\partial y}\right)^{2}+\left(\frac{\partial w^{\prime}}{\partial y}\right)^{2}\right] \\
& +\left(\frac{\sigma B_{0}^{2} \operatorname{Sin}^{2} \alpha}{1+m^{2} \operatorname{Sin}^{2} \alpha}-\frac{v^{\prime}}{k}\right)\left(u^{\prime 2}+w^{\prime 2}\right)
\end{aligned}
$$

Corresponding the initial and boundary conditions are

$$
\begin{gathered}
u^{\prime}=w^{\prime}=0 \text { at } t \leq 0, \\
u^{\prime}=w^{\prime}=0 \text { at } y=-1 \\
u^{\prime}=1, w^{\prime}=0 \text { at } y=1 \text { for } t>0, \\
\theta=0 \text { for } t \leq 0, \text { and } \theta=0 \text { at } y=-1, \theta=1 \\
\text { At } y=1 \text { for } t>0
\end{gathered}
$$

\section{III.NUMERICAL PROCEDURE}

Using a similar transformation, the differential equation is reduced to a common differential equation of higher order. In addition, these differential equations of higher order are solved numerically with integrated digital solver bvp4c from MATLAB.

\section{RESULTS AND DISCUSSION}

To find a numerical interpretation of a particular problem, a parametric study was conducted to demonstrate the effects of its control parameters: Bingham number, Reynolds number, Hartmann number, Prandtl number and Eckert number. Figure (1-12) shows the velocity component and temperature profile for a fixed time value or a Bingham number. In Figure (1-3)that increasing the elastic limit of the Bingham number lowers the velocity component and increases the viscosity as the time they reach the stable valve increases. These figures also show that the speed component does not reach its equilibrium state monotonously. This behavior is more pronounced for Bingham numbers of smaller parameters and for velocity components. Figure(3)shows that the monotonic temperature curve reaches a stable it increase temperature. Figure (4-6)describes the velocity components and temperature changes for different values of Hartmann parameters in the middle of the channel. 
In these figures, the velocity and temperature component decrease with increasing magnetic field strength throughout the fluid area.

Figures (10-12) illustrate the components of velocity and temperature changes over time in each of the Hall parameters. Figure (10) shows that as the Hall coefficient increases, the effective conductivity decreases and the velocity component increases, reducing the magnetic damping force of the velocity component .

The time in the velocity component reaches its stable increases as the Hall parameter increases and decreases as the Bingham number increases.

In Figure (11), the velocity component increases as the reverb parameter increases because the velocity component is the result of the reverb effect. Figure (10-12) shows that the effect of Hall parameters on temperature depends on time. The increase of the Hall parameter decreases the temperature in a short time and increases the temperature when it is high. This shows the temperature history as a function of time for Bingham numbers. it was also observed that for all values of the Hall parameter, increasing the Bingham number would reduce the temperature. In fact, increasing the Bingham number reduces the velocity component and its gradient, thereby reducing joule and viscous entire range of fluids, the speed and temperature components are displayed over time for the control parameters. In order to examine the accuracy and correctness of the solutions, the results of the time development of the velocity components $u^{1}$ and $w^{1}$ at the centre of the channel for the Newtonian case is compared and shown, as depicted Table 1, to have complete agreement with those reported by Attia . This ensures the satisfaction of all the governing equations; mass continuity, momentum and energy equations.

Table-1

\begin{tabular}{|c|c|c|c|c|c|c|}
\hline \multirow[t]{2}{*}{$t$} & \multicolumn{3}{|c|}{ The values of $u$ ' } & \multicolumn{3}{|c|}{ The values of $w$ ' } \\
\hline & $\begin{array}{c}\text { Prese } \\
\text { nt } \\
\text { Result } \\
\text { s } \\
\end{array}$ & Attia & Attia & $\begin{array}{c}\text { Prese } \\
\text { nt } \\
\text { Result } \\
\text { s }\end{array}$ & Attia & Attia \\
\hline $\begin{array}{c}0 . \\
1\end{array}$ & $\begin{array}{c}0.546 \\
2\end{array}$ & $\begin{array}{c}0.467 \\
3\end{array}$ & $\begin{array}{c}0.466 \\
9\end{array}$ & $\begin{array}{c}0.045 \\
6\end{array}$ & $\begin{array}{c}0.060 \\
3\end{array}$ & $\begin{array}{c}0.061 \\
9\end{array}$ \\
\hline 0. & 0.633 & 0.809 & 0.808 & 0.086 & 0.206 & 0.205 \\
\hline 2 & 5 & 4 & 9 & 6 & 0 & 6 \\
\hline $\begin{array}{l}0 . \\
3\end{array}$ & $\begin{array}{c}0.683 \\
4\end{array}$ & $\begin{array}{c}1.016 \\
5\end{array}$ & $\begin{array}{c}1.016 \\
0\end{array}$ & $\begin{array}{c}0.093 \\
3\end{array}$ & $\begin{array}{c}0.369 \\
2\end{array}$ & $\begin{array}{c}0.368 \\
7\end{array}$ \\
\hline $\begin{array}{l}0 . \\
4\end{array}$ & $\begin{array}{c}0.798 \\
8 \\
\end{array}$ & $\begin{array}{c}1.125 \\
4\end{array}$ & $\begin{array}{c}1.125 \\
1\end{array}$ & $\begin{array}{c}0.134 \\
5 \\
\end{array}$ & $\begin{array}{c}0.517 \\
7 \\
\end{array}$ & $\begin{array}{c}0.517 \\
1\end{array}$ \\
\hline $\begin{array}{l}0 . \\
5\end{array}$ & $\begin{array}{c}0.842 \\
8 \\
\end{array}$ & $\begin{array}{c}1.170 \\
9\end{array}$ & $\begin{array}{c}1.170 \\
8 \\
\end{array}$ & $\begin{array}{c}0.521 \\
1 \\
\end{array}$ & $\begin{array}{c}0.637 \\
5 \\
\end{array}$ & $\begin{array}{c}0.637 \\
0\end{array}$ \\
\hline $\begin{array}{l}0 . \\
6 \\
\end{array}$ & $\begin{array}{c}0.867 \\
4 \\
\end{array}$ & $\begin{array}{c}1.179 \\
1 \\
\end{array}$ & $\begin{array}{c}1.179 \\
1 \\
\end{array}$ & $\begin{array}{c}0.665 \\
2 \\
\end{array}$ & $\begin{array}{c}0.726 \\
4 \\
\end{array}$ & $\begin{array}{c}0.726 \\
0 \\
\end{array}$ \\
\hline $\begin{array}{l}0 . \\
7\end{array}$ & $\begin{array}{c}0.899 \\
4\end{array}$ & $\begin{array}{c}1.168 \\
1\end{array}$ & $\begin{array}{c}1.168 \\
2\end{array}$ & $\begin{array}{c}0.775 \\
6\end{array}$ & $\begin{array}{c}0.787 \\
6\end{array}$ & $\begin{array}{c}0.787 \\
2\end{array}$ \\
\hline $\begin{array}{l}0 . \\
8\end{array}$ & $\begin{array}{c}0.998 \\
2\end{array}$ & $\begin{array}{c}1.149 \\
4\end{array}$ & $\begin{array}{c}1.149 \\
5\end{array}$ & $\begin{array}{c}0.791 \\
5\end{array}$ & $\begin{array}{c}0.826 \\
6\end{array}$ & $\begin{array}{c}0.826 \\
3\end{array}$ \\
\hline $\begin{array}{l}0 . \\
9 \\
\end{array}$ & $\begin{array}{c}1.000 \\
0\end{array}$ & $\begin{array}{c}1.129 \\
5\end{array}$ & $\begin{array}{c}1.129 \\
7\end{array}$ & $\begin{array}{c}0.834 \\
1 \\
\end{array}$ & $\begin{array}{c}0.846 \\
6 \\
\end{array}$ & $\begin{array}{c}0.849 \\
1\end{array}$ \\
\hline $\begin{array}{l}1 . \\
0\end{array}$ & $\begin{array}{c}1.000 \\
1\end{array}$ & $\begin{array}{c}1.112 \\
0 \\
\end{array}$ & $\begin{array}{c}1.112 \\
2 \\
\end{array}$ & $\begin{array}{c}0.853 \\
5\end{array}$ & $\begin{array}{c}0.869 \\
3 \\
\end{array}$ & $\begin{array}{c}0.860 \\
7 \\
\end{array}$ \\
\hline
\end{tabular}

\section{CONCLUSION}

An unsteady magnetohydrodynamics flow of Bingham fluid with Permeable walls of Heat Transfer has studied in the current paper. The resulting higher order differential equations are solved using MATLAB with the integrated bvp4c digital solver for a given boundary condition. Here are some important conclusions from this review.

- Bingham number increases velocity components decreases at increasing the time.

- The velocity and temperature components decrease with increasing magnetic field strength.

- The Hall parameter increases and the temperature drop rapidly and increase rapidly.

- Bingham number increases temperature decreases for all values of hall parameter.

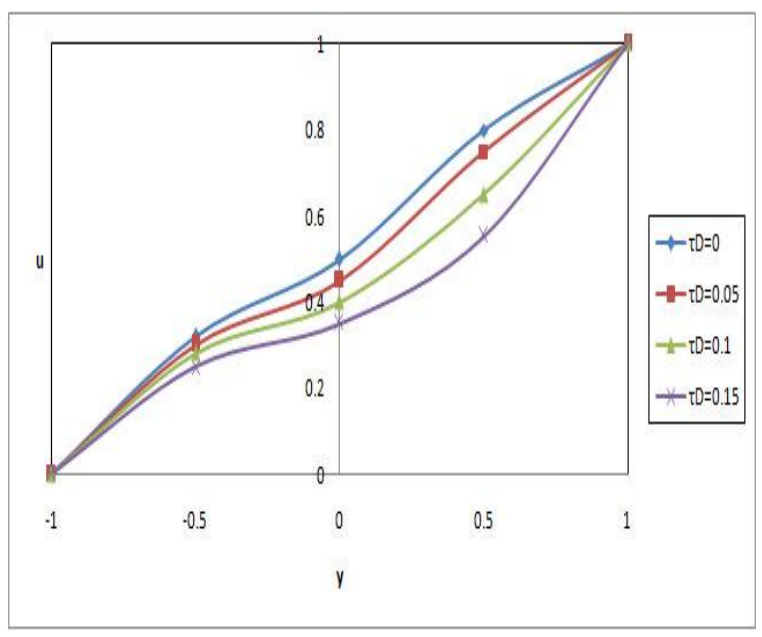

Figure 1

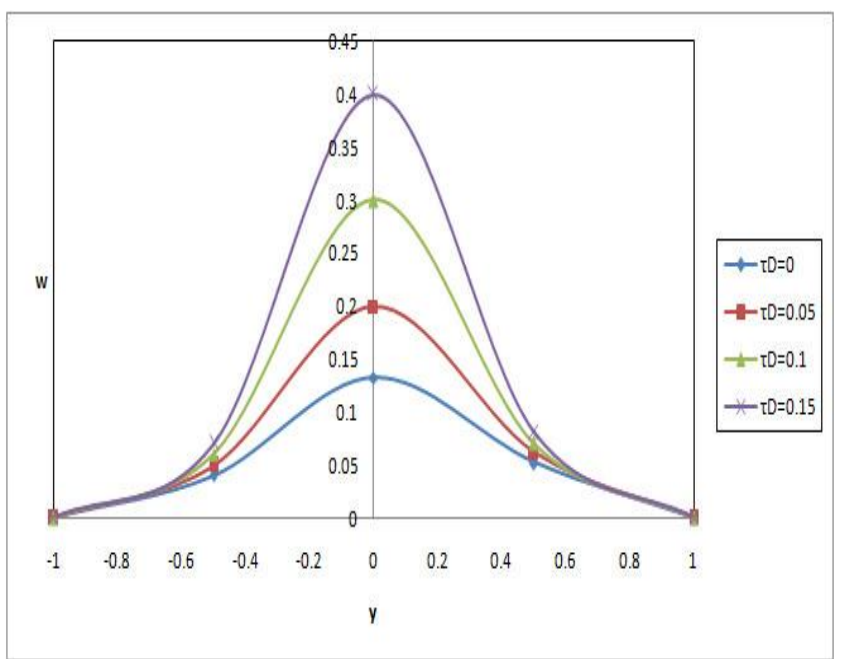

Figure 2 
An Unsteady Magnetohydrodynamics Flow of Bingham Fluid with Hall Effect of Heat Transfer

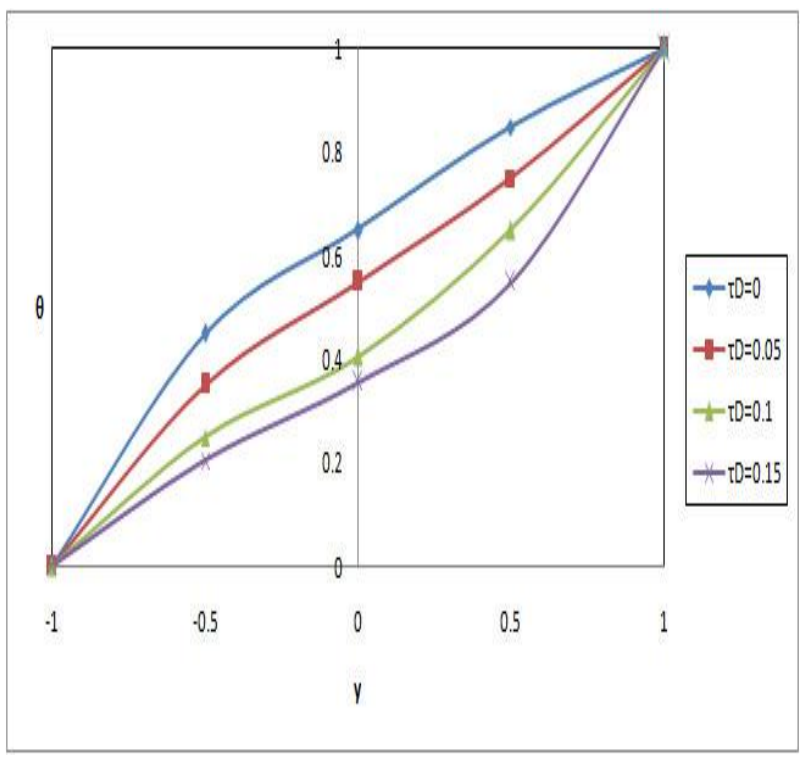

Figure 3

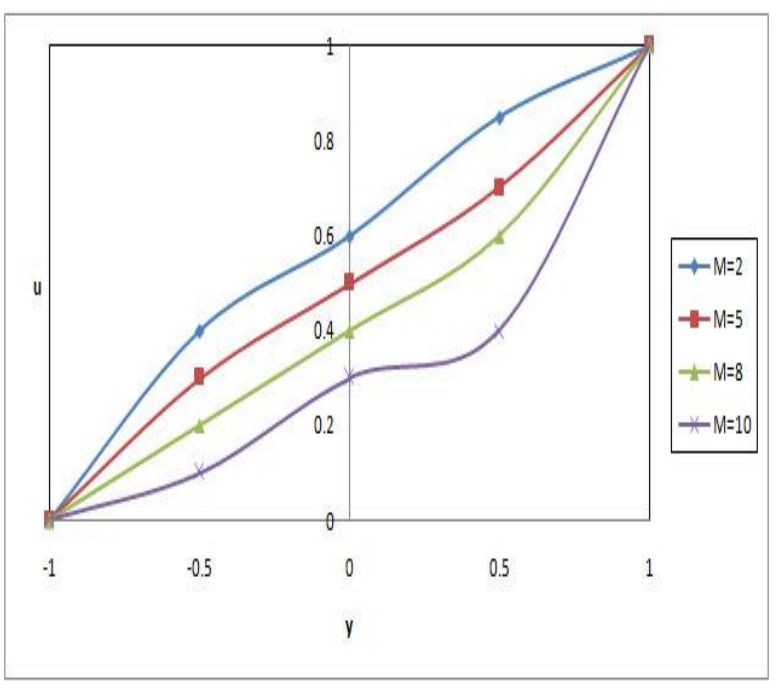

Figure 4

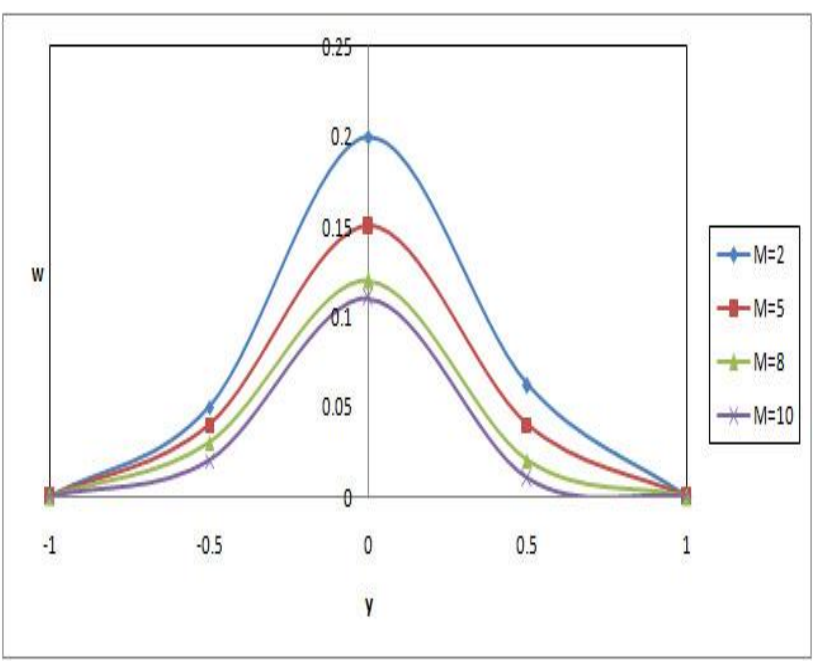

Figure 5

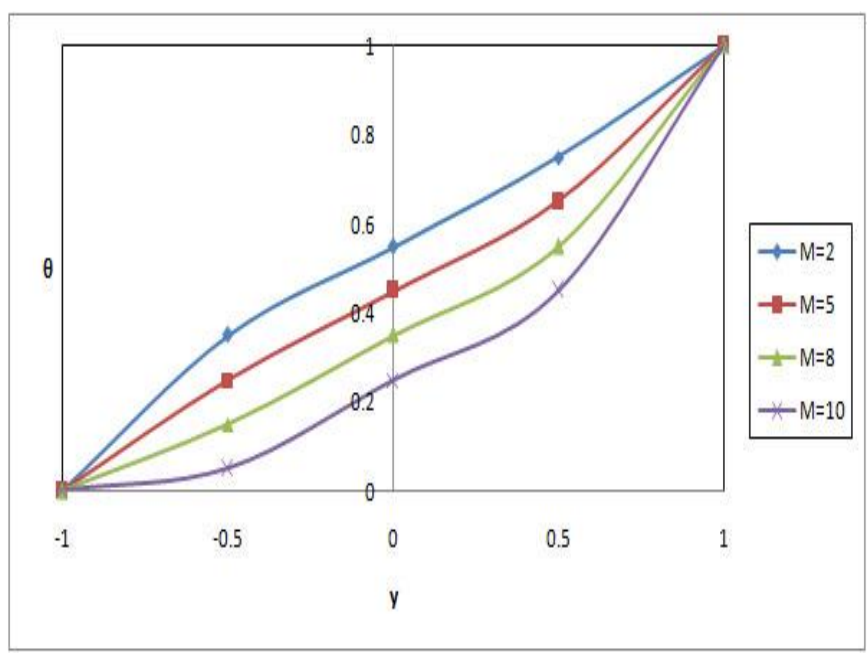

Figure 6

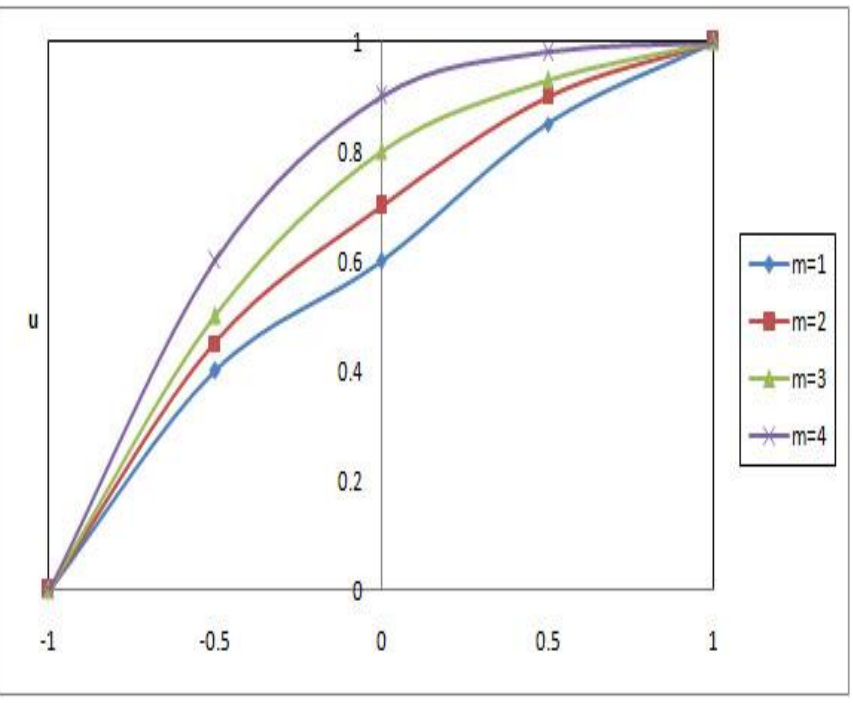

Figure 7

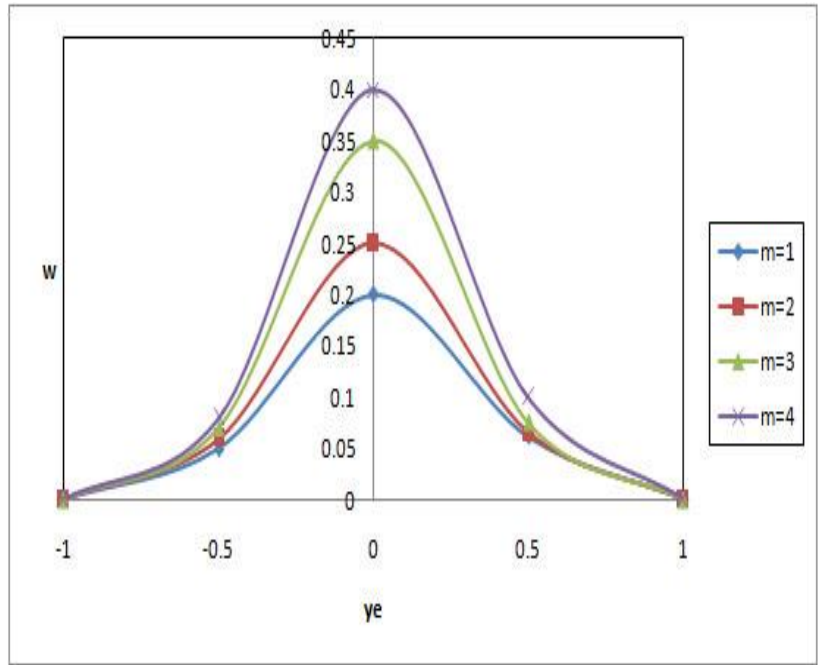

Figure 8 


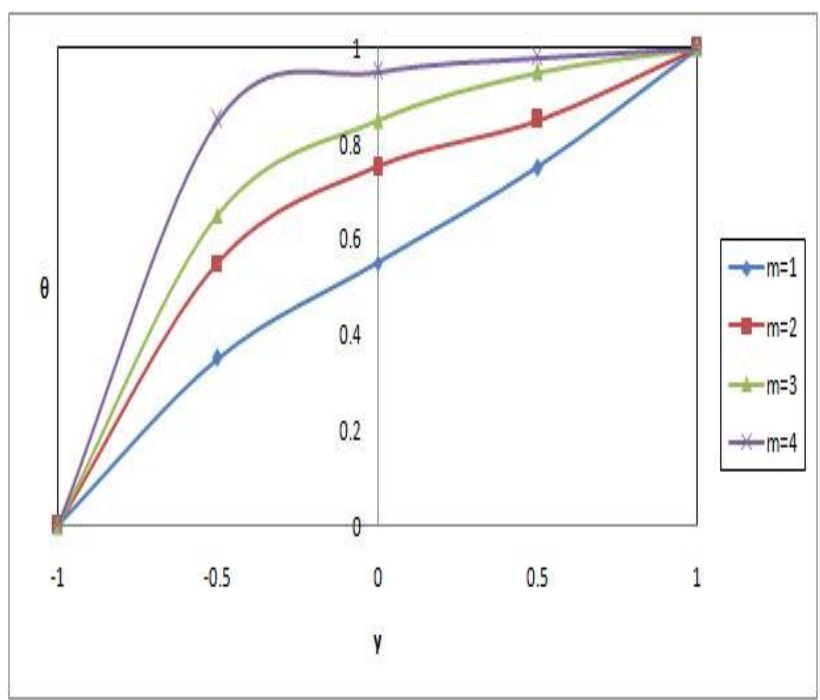

Figure 9

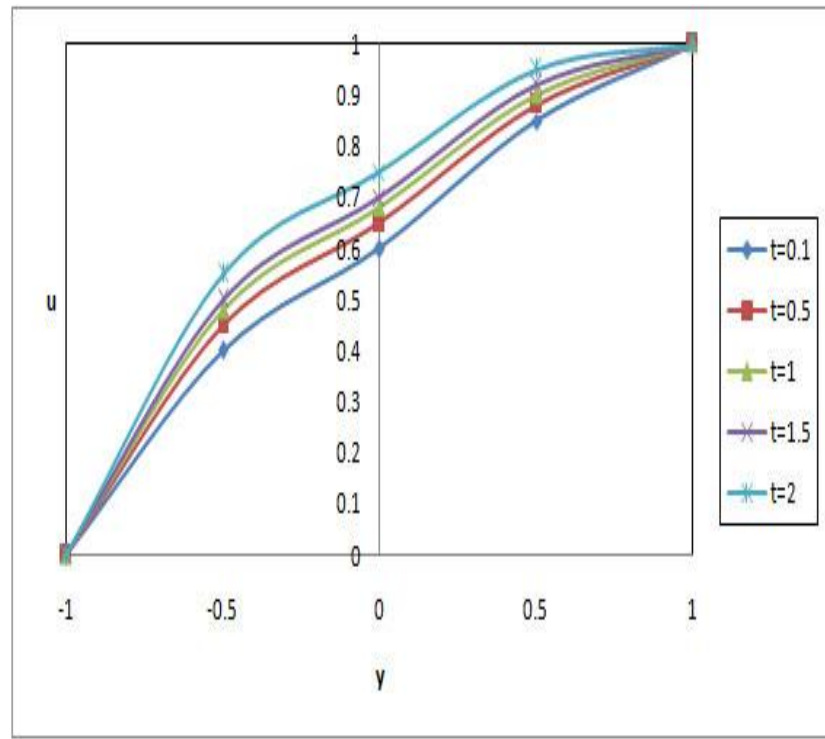

Figure 10

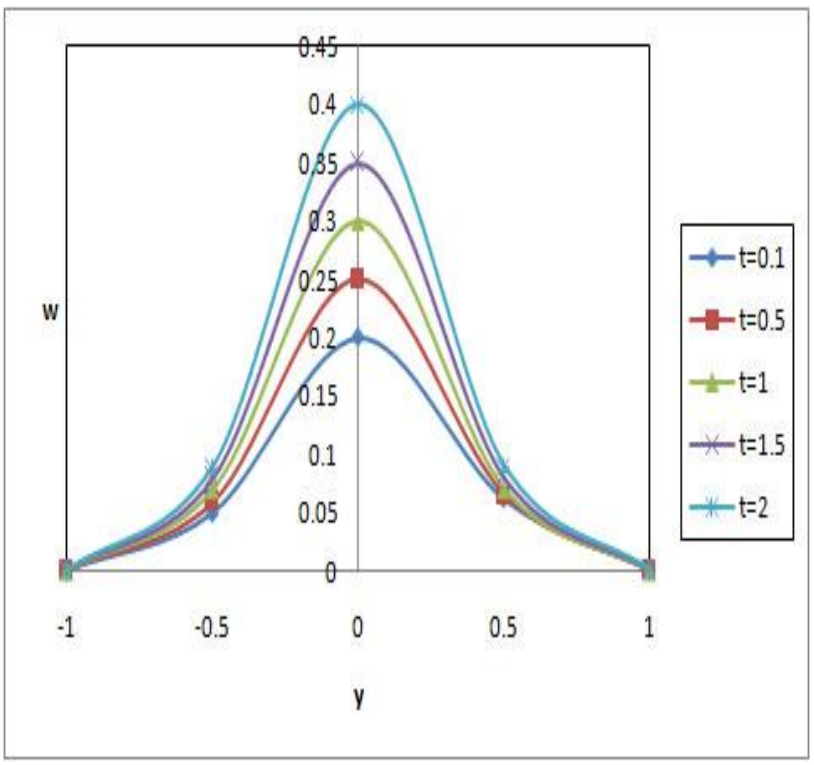

Figure 11

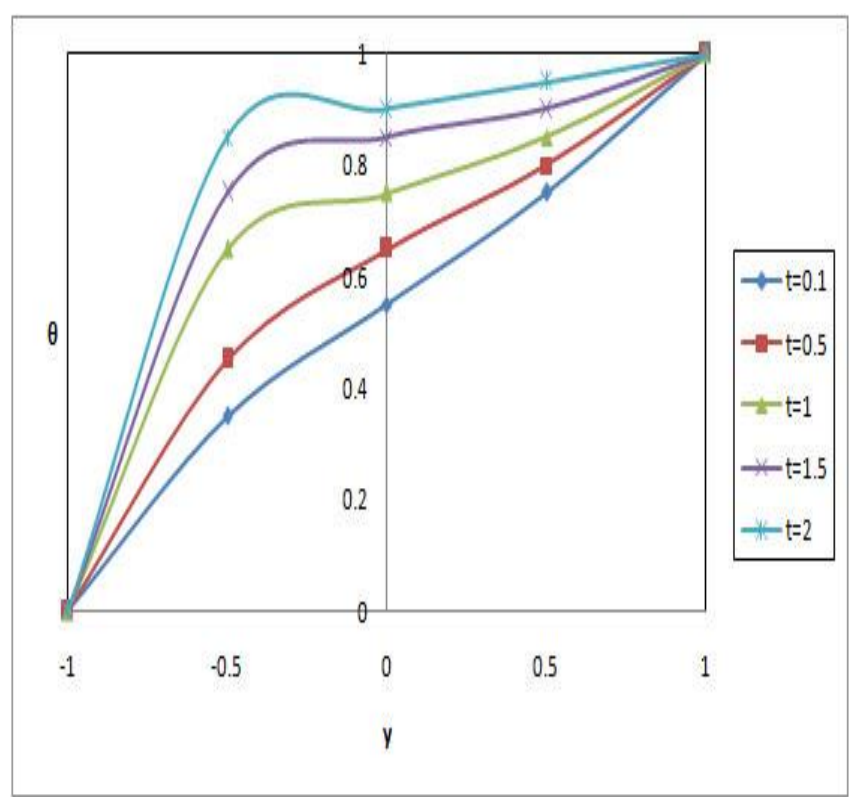

Figure 12

\section{REFERENCES:}

1. Nigam, Singh, Heat transfer blades between the axes in a near parallel transverse direction, Qutly. Journal of Mechanics and Applied Mathematics,1960, 13, 85.

2. Alpher, Heat transfer in the magnetic fluid flow between paralle plates, International Journal of Heat Mass Transfer, 1961, 3, 108.

3. Walters, K. Development of Non-Newtonian Fluid Mechanics Personal Perspective. J. Non-Newtonian Fluid Mechanics, 1979, 5, 113-124.

4. Astarita, G. Three alternative method for the development of constitutive equations. J. Non-Newtonian Fluid Mechanics, 1979 $5,125-140$

5. Walton and S.H. Bittleston, Bingham plastic axial flow in a tight eccentric ring, J. of Fluid Mech., 1991,222, 39-60.

6. Attia, Kotb, MHD transfers heat between two parallel plates, Acta Mechanics, 1996,117, 215-220.

7. Attia, Transient, MHD flow and heat transfer between two paralle plates with temperature dependent viscosity, Mechanics Research Communications, 1996, 26, 115-121.

8. Chamkha, Unsteady laminar flow of hydrogen-hydrogen fluid particles and heat transfer in channels and tubes, Internal J. Heat Fluid Flow, 2000, 21, 740-746.

9. Attia, Unsteady MHD flow between parallel plates with variable physical properties and heat transfer from dusty fluids, Applied Mathematical Modeling, 2002, 13, 863-875.

10. Hazem Ali Attia and M.E.Sayed Ahmed., Hall effects the flow of heat transfer of a certain wave effect on small particles into the fluid Bingham, Applied Mathematical Modelling, 2004, 28, 1027 1045.

11. Chamkha A J, Unsteady MHD convective heat transfer and mass transfer through semi-vertical permeable moving plates, heat transfer and mass transfer through semi-vertical and endothermic diathermy plates, International journal of Engineering Science, 2004, 42, 217-230.

12. Ogulu, Amakiri and Mbeledogu, Heat transfer by radiation, the compressed fluid flow without MHD means that the plate is moving vertically due to unstable convection, International Journal of Heat and Mass Transfer,2007, 5,1668-1674.

13. Ramachandra Prasad and Bhaskar Reddy, Effect of radiation on convective heat and unstable MHD mass transfer in

a mobile semi-infinite vertical transmission plate embedded in a porous medium, J. of Energy Heat and Mass Transfer, 2008, 30, 57-68.

14. Dass SS, Satapathy JK, Panda JP. Under the influence of vibration absorption and a heat source, the effect of mass and heat transfer from MHD to MHD through a porous medium through vertical porous plates. Inl. J. of Heat Mass Transfer, 2009, 52, 5962-5969.

15. Ahmed ,Khatun ,Oscillating magnetohydrodynamic flow in porous flat channels with suction and injection. Internal Journa Engineering Technology, 2013, 11, 1024-1029. 
16. Makinde, Chinyoka, Rundora, Variable viscosity non-Newtonian fluid in porous saturated media with asymmetric convective boundary conditions, Comp. Math. Appli., 2011, 62, 3343-3352.

17. Rundora , Makinde. Influence of suction / injection on a nonNewtonian fluid flow having a reactive variable viscosity in a transient state and conditions of convection limits in channels filled with a porous medium, Journal of Petroleum Science and Engineering, 2013,108, 328-35.

18. Chuo-Jeng Huang, The effect of uniform air blowing / breathing on thermal radiation in porous media and the free convection of non-Newtonian fluids on vertical conical surfaces under the influence of the Soret / Dufour effect: uniform wall temperature / uniform wall thickness, 2016, 139, 032501-032508.

\section{AUTHORS PROFILE}

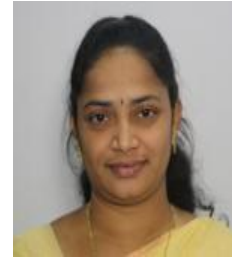

Ms. Pudhari Srilatha, is working as an Associate professor of mathematics at Institute of Aeronautical Engineering, An autonomous engineering college at Hyderabad. She has 12 years of wide experience in teaching. She has published 11 research papers in her thrust area of research -fluid dynamics.

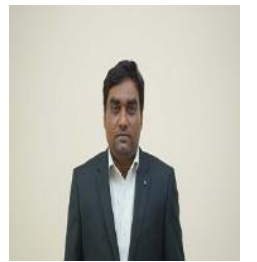

Mr. J Suresh Goud, is working as an Associate professor of mathematics at Institute of Aeronautical Engineering, at Hyderabad. He has 12 years of wide experience in teaching. He has published 13 research papers in national and international journals in his thrust area of research -fluid dynamics.

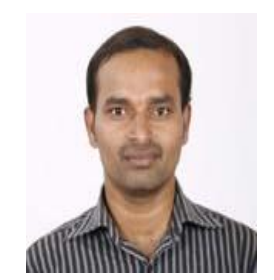

Mr. B Raju, is working as an Assistant professor at Institute of Aeronautical Engineering, Hyderabad. He has 12 years of wide experience in teaching. $\mathrm{He}$ has published 4 research papers.

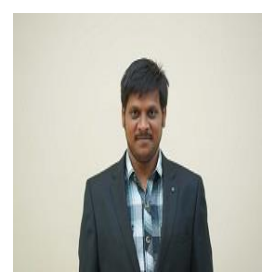

Mr. S Devaraj is an Assistant professor in Department of Aeronautical Engineering at Institute of Aeronautical Engineering, Hyderabad. He has completed his M.Tech from JNTUH. He has 10 years of teaching experience. He has published 4 research papers in national and international journals. 